\title{
Nonlinear Photoemission Microscopy with Surface Plasmon Polaritons
}

\author{
F.-J. Meyer zu Heringdorf and N. M. Buckanie \\ * Department of Physics and Center for Nanointegration Duisburg-Essen (CeNIDE), University of \\ Duisburg-Essen, Lotharstrasse 1, 47057 Duisburg, Germany
}

During the last years it has become evident that photoemission electron microscopy (PEEM) is well suited for studying the dynamics of electronic excitations at surfaces with spatial resolution, using fs laser pulses for excitation and probing. As long as the photon energy of the used fs laser pulses $(\mathrm{E} \sim 3.1 \mathrm{eV})$ is lower than the ionization potential of the object of interest, photoemission must proceed via two photon photoemission (2PPE) either through a virtual or a real intermediate state, the dynamics of which can be analyzed in pump-probe experiments. In our earlier work [1] we demonstrated that plasmon resonances (LSPs) in small Ag islands can act as intermediate states for 2PPE and result in enhanced photoemission yields. Recently it was shown that Surface Plasmon Polariton (SPP) waves can be imaged with PEEM as well [2, 3]. The contrast mechanism for imaging SPPs in 2PPE is, however, more complicated. If a Ag nanowire is illuminated with $\lambda=400 \mathrm{~nm}$ fs laser pulses, the excitation conditions for the SPP wave dictate that a SPP wave can only be started at the edge of the wire due to the momentum vector necessary to couple light into the SPP. This situation is sketched in Fig. 1 (a). After excitation, the SPP wave propagates along the surface of the wire. Because of the finite dimensions of the exciting laser pulses and the grazing incidence geometry, however, the laser pulse propagates along the surface as well. PEEM detects the temporal integral of the fourth power of the superposed electric fields of the laser pulse and the SPP wave at a given location [3].

For a nanowire like in Fig. 1(a), the result of the superposition is the slow varying component of a beat pattern that is formed between the laser and the SPP wave. The pattern exhibits a periodicity of $\sim 2.5 \mu \mathrm{m}$ as can be calculated from the dielectric function of the Ag nanowire, the wavelength of the laser pulses, and their angle of incidence relative to the surface [2]. While in the simple 1D case of a Ag nanowire the SPP wave and the laser propagate in the same direction, this is not necessarily true when extended 2D islands are investigated. Fig. 1 (b) shows a rather flat triangular Ag island that has self-assembled on a Si(111) surface. Fig. 1 (c) shows the same island in 2PPE PEEM, using $\lambda=400 \mathrm{~nm}$ fs laser pulses for illumination. The incidence direction of the laser pulses is from the lower left, under a grazing angle of $74^{\circ}$ with respect to the surface normal. The SPP related pattern on top of the island is obviously more complicated than what the 1D case would suggest. First, there are two SPP waves involved that are started at two of the edges of the island, labeled $\mathrm{SPP}_{1}$ and $\mathrm{SPP}_{2}$. Second, the 2PPE patterns of the two SPP waves are aligned with the edges of the triangular island, suggesting that the propagation direction of the SPP wave is normal to the islands' edge. Interestingly, this turns out to be not correct.

The $2 \mathrm{D}$ analogon of a $1 \mathrm{D}$ beat pattern is a moiré pattern. In Fig. 1 (d) the situation of $\mathrm{SPP}_{1}$ of Fig. 1 (c) is illustrated. The in-plane component of the light wave points in direction of $\mathbf{k}_{\text {light }}$, and the resulting $\mathrm{SPP}_{1}$ wave of slightly smaller wavelength [2] propagates in a direction $\mathbf{k}_{\mathrm{SP} 1}$ that is in this case rotated clockwise relative to $\mathbf{k}_{\text {light }}$. As a result of the superposition of the two waves a moiré pattern in direction $\mathbf{k}_{\text {moire }}$ is formed that resembles the experimentally observed fringes on the left side of the Ag island in Fig. 1 (c). The orientation and the fringe spacing of the moiré pattern can be easily calculated [4] and they depend on the wavelength of the two involved waves and the angle between them. If $\mathbf{k}_{\text {light }}$ and $\mathbf{k}_{\mathrm{SPP} 1}$ enclose the angle $2 \alpha$, a moiré pattern is formed under the angle $\theta+\alpha$ 
with respect to the light wave. Clearly, the orientation of the moire pattern, $\mathbf{k}_{\text {moire, }}$ is different than the propagation direction $\mathbf{k}_{\mathrm{SPP} 1}$ of the $\mathrm{SPP}_{1}$ wave. The naïve interpretation of the moire pattern and its suggested propagation direction of the SPP wave are thus counterintuitive. Only a thorough analysis of the moiré pattern, with exact knowledge of the incidence geometry of the laser pulses, allows a reconstruction of the propagation direction of the SPP wave from the moire pattern. Such analysis reveals that the light wave and the SPP wave fulfill Snell's law of refraction. It can easily be shown that the fulfillment of this condition always causes the moire pattern to be aligned with the islands' edge, independent of its orientation relative to $\mathbf{k}_{\text {light }}$ [5].

(a) Laser SPP Interaction

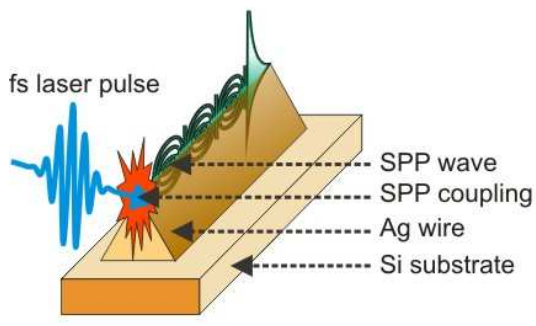

(c) 2PPE PEEM

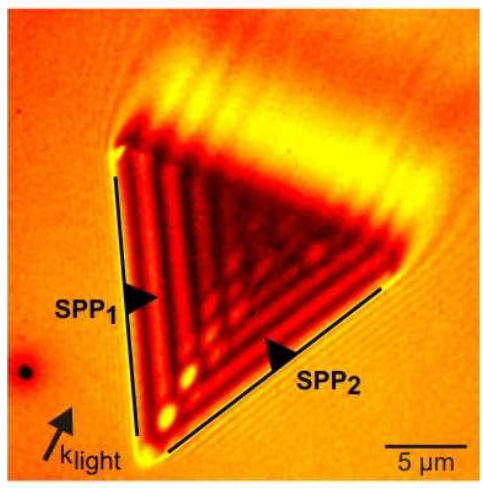

(b) Ag Island in 1PPE (Hg Lamp)

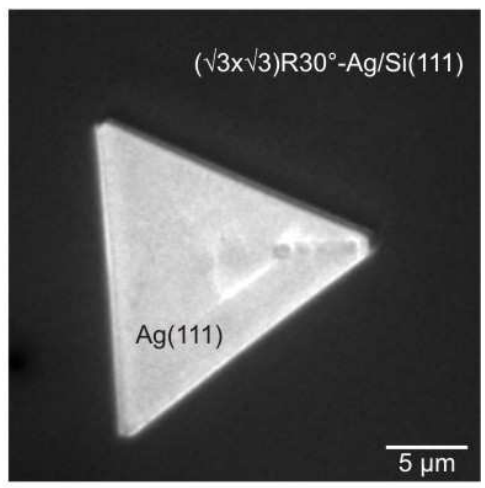

(d) Moiré Angles

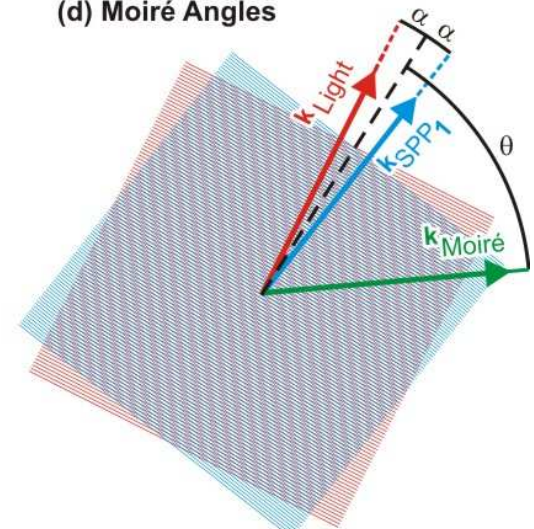

FIG 1. (a) Illustration of SPP contrast formation in 2PPE PEEM for a Ag nanowire; (b) Threshold PEEM image of a triangular Ag island on Si(111); (c) 2PPE PEEM image of the same island; (d) definition of angles for the moiré pattern formation.

\section{References}

[1] L. Chelaru et al., Phys. Rev. B 73 (2006) 115416

[2] L. Chelaru et al., Surf. Sci. 601 (2007) 4541

[3] A. Kubo et al., Nano Lett. 7 (2007) 470

[4] K. Creath and J. Wyan, Optical Shop Testing, Wiley (1992).

[5] This work was supported by the Deutsche Forschungsgemeinschaft through the collaborative research center SFB 616 "Energy Dissipation at Surfaces" and the priority programme SPP 1391 "Ultrafast Nanooptics". 